\title{
Eventually Pointed Principally Ordered Regular Semigroups
}

\author{
G.A. Pinto
}

Department of Mathematics, Sultan Qaboos University, P.O. Box 36, PC 123, Al- Khoud, Muscat Sultanate of Oman. Email: goncalo@squ.edu.om.

\begin{abstract}
An ordered regular semigroup, $S$, is said to be principally ordered if for every $x \in S$ there exists $x^{*}=\max \{y \in S \mid x y x \leq x\}$. A principally ordered regular semigroup is pointed if for every element, $x$, we have $x^{2} \leq x$. Here we investigate those principally ordered regular semigroups that are eventually pointed in the sense that for all $x \in S$ there exists a positive integer, $n$, such that $\left(x^{n}\right)^{2} \leq x^{n}$. Necessary and sufficient conditions for an eventually pointed principally ordered regular semigroup to be naturally ordered and to be completely simple are obtained. We describe the subalgebra of $\left(S,{ }^{*}\right)$ generated by a pair of comparable idempotents $e$ and $f$ such that $e^{0}=f^{0}$.
\end{abstract}

Keywords: Regular semigroup; Strong Dubreil-Jacotin; Principally ordered; Naturally ordered; Pointed principally ordered; Green's relations; Completely simple.

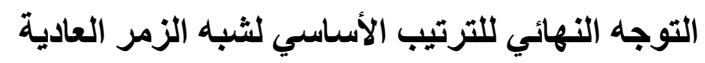

$$
\text { ج.أ بينتو }
$$

الملخص: يقال إن شبه الزمر العادية المرتبة،

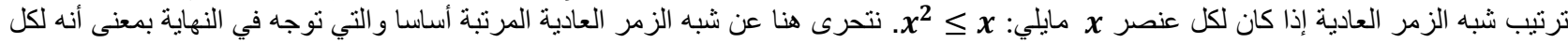

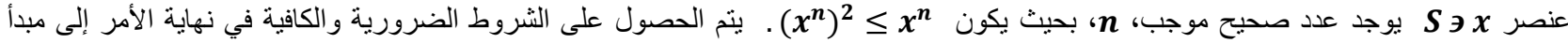

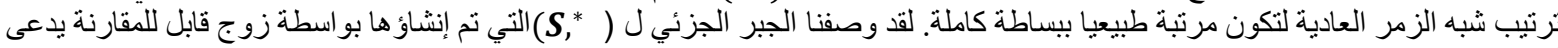
أيديمبوتنتسو يكون.

الكلمات المفتاحية: شبه الزمر العادية، دوبريل-جاكوتن القوي، مرتب أساسا، مرتب طبيعيا، مرتب وموجه أساسا، علاقات جرين، بسيط متكامل.

\section{Introduction}

A $\mathrm{n}$ ordered regular semigroup, $S$, is said to be principally ordered if for every $x \in S$ there exists $\mathbf{A}_{x^{*}}=\max \{y \in S \mid x y x \leq x\}$. The basic properties of the unary operation $x \rightarrow x^{*}$ in such semigroups were established in [1] and [2] and are listed in [3, Theorem 13.26]. In particular, we recall for the reader's convenience that, in such a semigroup, the following properties hold, and will be used throughout in what follows:

$\left(P_{1}\right)(\forall x \in S) \quad x=x x^{*} x$

$\left(P_{2}\right)$ every $x \in S$ has a biggest inverse, namely $x^{0}=x^{*} x x^{*}$

$\left(P_{3}\right)(\forall x \in S) \quad x^{0} \leq x^{*}$

$\left(P_{4}\right)(\forall x \in S) \quad x \leq x^{* *}$

In [4] we introduced the class of pointed principally ordered regular semigroups. We say that a principally ordered regular semigroup, $S$, is pointed, if the classes modulo Green's relations $\mathcal{R}, \mathcal{L}, \mathcal{D}$ have biggest elements which are idempotent. In [4, Theorem 1] we proved, in particular, that a principally ordered regular semigroup $S$ is pointed if and only if $x^{2} \leq x$, for every $x \in S$. In this paper we investigate a generalization of pointed principally ordered regular semigroups.

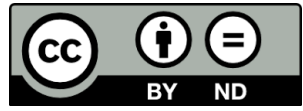




\section{Eventually pointed principally ordered regular semigroups}

Definition Let $S$ be a principally ordered regular semigroup. We say that $S$ is eventually pointed if, for every $x \in S$, there exists a positive integer, $n$, such that $\left(x^{n}\right)^{2} \leq x^{n}$, that is, $x^{2 n} \leq x^{n}$.

An immediate observation is that a pointed principally ordered regular semigroup is eventually pointed. Simply take $n=1$.

Note that any finite ordered regular semigroup, $S$, is eventually pointed. In fact, there exists a positive integer power of each element $S$ that is an idempotent, and $S$ is eventually pointed.

Under the notation of the previous definition, we have that

$$
x^{n} \cdot x^{n} \cdot x^{n}=x^{2 n} \cdot x^{n} \leq x^{n} \cdot x^{n}=x^{2 n} \leq x^{n}
$$

from which we may conclude the following property

$(\alpha)(\forall x \in S)\left(\exists n \in \mathbb{Z}^{+}\right) \quad x^{n} \leq\left(x^{n}\right)^{*}$

Now, let us present examples that show that we are, in fact, in the presence of a new class of semigroups.

Example 1. Consider $\mathrm{Mat}_{2} B$, the ordered regular semigroup of $2 \times 2$ matrices with entries in a Boolean algebra $B$, and matrix multiplication, which can be seen in [1, Example 13.1]). For the basic operations in $B$ we use the notation $a+b$ (for $a \vee b$ ) and $a b$ (for $a \wedge b$ ). The subset $\operatorname{Mat}_{2} B(0,1)$ where all the entries of the matrices are either 0 or 1 , respectively the bottom and top element of $B$, is a semigroup for matrix multiplication. Routine calculations allow us to say that $\mathrm{Mat}_{2} B(0,1)$ is a principally ordered regular semigroup with Hasse diagram:

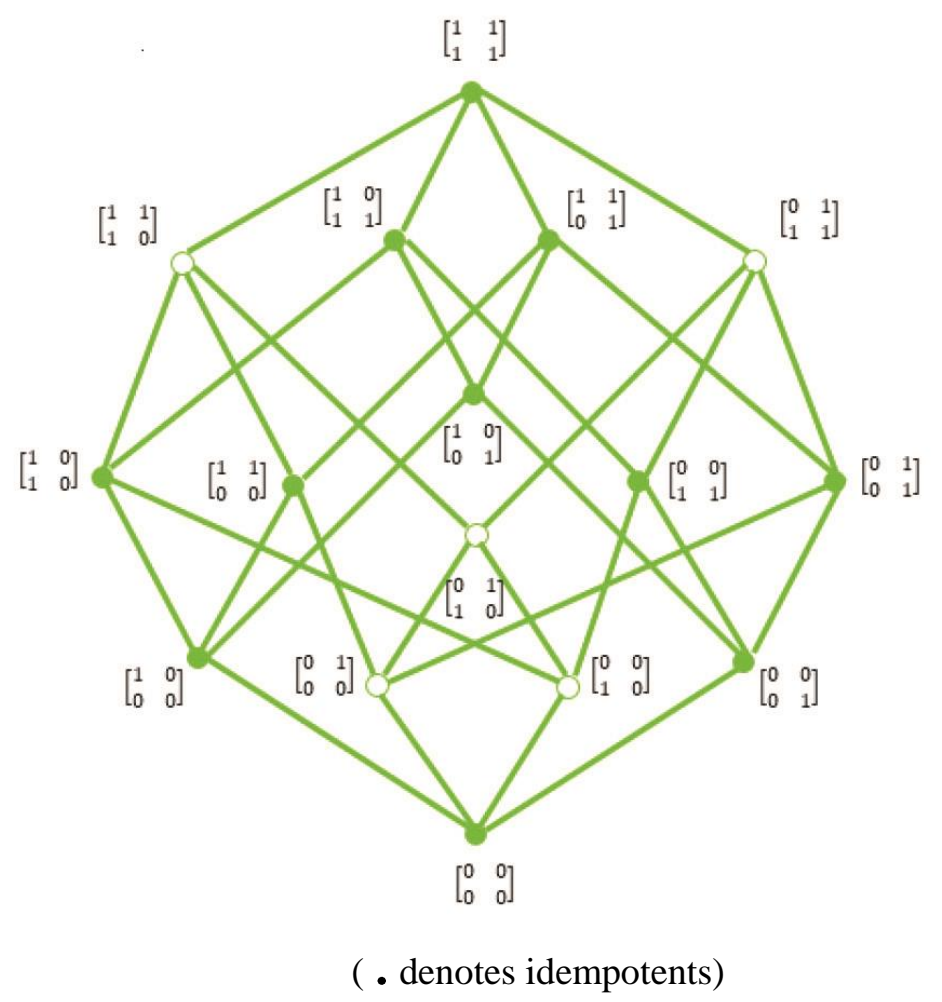

where

$$
\left[\begin{array}{ll}
0 & 1 \\
1 & 0
\end{array}\right]^{*}=\left[\begin{array}{ll}
0 & 1 \\
1 & 0
\end{array}\right], \quad\left[\begin{array}{ll}
1 & 0 \\
0 & 1
\end{array}\right]^{*}=\left[\begin{array}{ll}
1 & 0 \\
0 & 1
\end{array}\right], \quad\left[\begin{array}{ll}
1 & 0 \\
1 & 1
\end{array}\right]^{*}=\left[\begin{array}{ll}
1 & 0 \\
1 & 1
\end{array}\right]
$$

and

$$
\left[\begin{array}{ll}
1 & 1 \\
0 & 1
\end{array}\right]^{*}=\left[\begin{array}{ll}
1 & 1 \\
0 & 1
\end{array}\right], \quad\left[\begin{array}{ll}
1 & 1 \\
1 & 0
\end{array}\right]^{*}=\left[\begin{array}{ll}
0 & 1 \\
1 & 1
\end{array}\right], \quad\left[\begin{array}{ll}
0 & 1 \\
1 & 1
\end{array}\right]^{*}=\left[\begin{array}{ll}
1 & 1 \\
1 & 0
\end{array}\right]
$$




\section{EVENTUALLY POINTED PRINCIPALLY ORDERED REGULAR SEMIGROUPS}

and, for every other element $\left[\begin{array}{ll}a & b \\ c & d\end{array}\right] \in \operatorname{Mat}_{2} B(0,1)$,

For biggest inverses

$$
\left[\begin{array}{ll}
a & b \\
c & d
\end{array}\right]^{*}=\left[\begin{array}{ll}
1 & 1 \\
1 & 1
\end{array}\right]
$$

and for any other element in $\left[\begin{array}{ll}a & b \\ c & d\end{array}\right] \in \operatorname{Mat}_{2} B(0,1)$,

$$
\left[\begin{array}{ll}
0 & 0 \\
0 & 0
\end{array}\right]^{0}=\left[\begin{array}{ll}
0 & 0 \\
0 & 0
\end{array}\right]
$$

$$
\left[\begin{array}{ll}
a & b \\
c & d
\end{array}\right]^{0}=\left[\begin{array}{ll}
a & b \\
c & d
\end{array}\right]^{*}
$$

Note that the element $\left[\begin{array}{ll}1 & 1 \\ 1 & 0\end{array}\right]$ in $\operatorname{Mat}_{2} B(0,1)$ is such that

$$
\left[\begin{array}{ll}
1 & 1 \\
1 & 0
\end{array}\right]^{2}=\left[\begin{array}{ll}
1 & 1 \\
1 & 0
\end{array}\right]\left[\begin{array}{ll}
1 & 1 \\
1 & 0
\end{array}\right]=\left[\begin{array}{ll}
1 & 1 \\
1 & 1
\end{array}\right]>\left[\begin{array}{ll}
1 & 1 \\
1 & 0
\end{array}\right]
$$

meaning that this principally ordered regular semigroup is not pointed. However, since it is a finite semigroup, it is eventually pointed.

Example 2. Consider the set $S=\{0, a, 1, b\}$ with the following Cayley table and Hasse diagram:

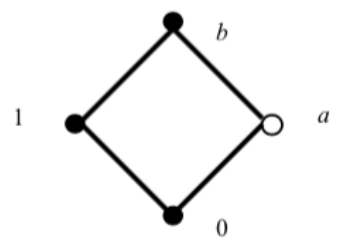

\begin{tabular}{c|cccc} 
& 0 & $a$ & 1 & $b$ \\
\hline 0 & 0 & 0 & 0 & 0 \\
$a$ & 0 & 1 & $a$ & $b$ \\
1 & 0 & $a$ & 1 & $b$ \\
$b$ & 0 & $b$ & $b$ & $b$
\end{tabular}

Routine calculations allow us to conclude that, with the multiplication and partial order just defined, $S$ is an ordered regular semigroup. In fact, it is a principally ordered semigroup, with

$$
0^{*}=b, 1^{*}=1, \quad b^{*}=b \quad \text { and } \quad a^{*}=a .
$$

Note that $S$ is not pointed since $a^{2}=1$ is not comparable with $a$. As in the previous example, we can conclude that, since $S$ is a finite semigroup, $S$ is an eventually pointed semigroup.

We can obtain this semigroup as a subsemigroup of $\operatorname{Mat}_{2} B(0,1)$, in Example 1. In fact,

$$
T=\left\{\left[\begin{array}{ll}
0 & 0 \\
0 & 0
\end{array}\right],\left[\begin{array}{ll}
0 & 1 \\
1 & 0
\end{array}\right],\left[\begin{array}{ll}
1 & 0 \\
0 & 1
\end{array}\right],\left[\begin{array}{ll}
1 & 1 \\
1 & 1
\end{array}\right]\right\}
$$

has the same Cayley table and Hasse diagram, denoting elements of $T$ respectively, by $0, a, 1, b$. Alternatively, if we take a Boolean Algebra, $B$, and any element in it, $x$, different from 0 and 1 , then

$$
U=\left\{\left[\begin{array}{ll}
0 & 0 \\
0 & 0
\end{array}\right],\left[\begin{array}{ll}
0 & x \\
x & 0
\end{array}\right],\left[\begin{array}{ll}
x & 0 \\
0 & x
\end{array}\right],\left[\begin{array}{ll}
x & x \\
x & x
\end{array}\right]\right\}
$$

with matrix multiplication and the Cartesian order, we have the same Cayley table and Hasse diagram, and therefore the same ordered semigroup.

Example 3. Consider the subset

$$
G=\left\{\left[\begin{array}{ll}
0 & 1 \\
1 & 0
\end{array}\right],\left[\begin{array}{ll}
1 & 0 \\
0 & 1
\end{array}\right]\right\}
$$

of $\mathrm{Mat}_{2} B(0,1)$ described in Example 1 . Obviously, it is a totally unordered group:

$\begin{array}{ll}01 & 10 \\ 10 & 01 \\ 0 & \bullet\end{array}$




\section{PINTO, G.A.}

We have that $\left[\begin{array}{ll}0 & 1 \\ 1 & 0\end{array}\right]^{*}=\left[\begin{array}{ll}0 & 1 \\ 1 & 0\end{array}\right]$ and $\left[\begin{array}{ll}1 & 0 \\ 0 & 1\end{array}\right]^{*}=\left[\begin{array}{ll}1 & 0 \\ 0 & 1\end{array}\right]$, meaning that $G$ is principally ordered.

However, it is not pointed since $\left[\begin{array}{ll}0 & 1 \\ 1 & 0\end{array}\right]^{2}=\left[\begin{array}{ll}1 & 0 \\ 0 & 1\end{array}\right]$ is not comparable with $\left[\begin{array}{ll}0 & 1 \\ 1 & 0\end{array}\right]$. By a similar argument to the one used in Example 1, we conclude that $G$ is eventually pointed.

Example 4. Consider the principally ordered band, constructed in [4, Example 1], $L^{[2]}=\{(x, y) \in L \times L \mid y \leq x\}$, where $L$ is a lattice and the multiplication is defined by

$$
(x, y)(a, b)=(x \vee a, y \wedge b)
$$

The Cartesian product with the Cartesian order of $L^{[2]}$ and $S$ in Example 2 is an infinite principally ordered regular semigroup that is eventually pointed, but not pointed.

Example 3 above provides an example of a principally ordered regular semigroup which is eventually pointed, that has a subgroup which is totally unordered and whose elements have finite order (one and two). This is always the case, as we can see in the next result, due to T. S. Blyth.

Theorem 1. If $S$ is an eventually pointed principally ordered regular semigroup and if $G$ is any subgroup of $S$, then $G$ is totally unordered and every $x \in G$ is of finite order.

Proof. For every $x \in G$ there exists a positive integer, $n$, such that $x^{2 n} \leq x^{n}$. Let $G^{+}=\left\{x \in G \mid 1_{G} \leq x\right\}$ be the positive cone of $G$. If $x \in G^{+}$, then we have that $1_{G} \leq x \leq x^{2} \leq \cdots \leq x^{n} \leq \cdots \leq x^{2 n} \leq \cdots$ whence $x^{n}=x^{2 n}$ and so $x^{n}$ is an idempotent in $G$. Thus $x^{n}=1_{G}$, consequently $x=1_{G}$ and $G^{+}=\left\{1_{G}\right\}$. The order in $G$ is then given by

$$
g \leq h \Leftrightarrow 1_{G} \leq h g^{-1} \Leftrightarrow h g^{-1} \in G^{+}=\left\{1_{G}\right\} \quad \Leftrightarrow \quad h=g .
$$

Thus, $G$ is totally unordered. Then, for every $x \in G$, it follows from $x^{2 n} \leq x^{n}$, that $x^{2 n}=x^{n}$ whence $x^{n}=1_{G}$ and so $x$ is of finite order.

Several of the results in [4], or similar ones, can be obtained in this new class of eventually pointed principally ordered regular semigroups. For this reason, it may be necessary to provide a new proof, or adjust the existent one for pointed principally ordered regular semigroups.

Theorem 2. Let $S$ be an eventually pointed principally ordered regular semigroup. If e is a maximal idempotent of $S$, then $e$ is a maximal element of $S$.

Proof. Let $e$ be a maximal idempotent of $S$ and consider any element $x$ in $S$ such that $e \leq x$. Since, $S$ is eventually pointed, there exists a positive integer, $n$, such that $x^{2 n} \leq x^{n}$. Then, $e=e^{n}=e \ldots e \leq x \ldots x=x^{n}$, and using $(\alpha)$, $e=e e \leq x^{n} x^{n} \leq x^{n}\left(x^{n}\right)^{*} \in E(S)$, whence $e=x^{n}\left(x^{n}\right)^{*}$. Now,

$$
x^{n}=x^{n}\left(x^{n}\right)^{*} x^{n}=e x^{n} \leq x x^{n}=x^{n+1} \leq \cdots \leq x^{2 n} \leq x^{n}
$$

from which we conclude that $x^{n} \in E(S)$ and consequently $e=x^{n}$. It follows that $e x=x^{n} x=x^{n+1}=x^{n}=e$, and similarly $x e=e$. Thus, $x e x=e x=e \leq x$ gives $e \leq x^{*}$, whence $e=e e \leq x x^{*}$, which implies $e=x x^{*}$ and therefore $x=x x^{*} x=e x=e$, meaning that $e$ is a maximal element of $S$.

Theorem 3. An eventually pointed principally ordered regular semigroup, $S$, has at most one maximal idempotent. Proof. Let $e, f$ be maximal idempotents of $S$. Consider the element $e f$ in $S$. Since $S$ is eventually pointed there exists a positive integer, $n$, such that $(e f)^{2 n} \leq(e f)^{n}$. From

$$
(e f)^{n} e(e f)^{n}=(e f)^{n} e e f(e f)^{n-1}=(e f)^{n} e f(e f)^{n-1}=(e f)^{2 n} \leq(e f)^{n}
$$

we obtain $e \leq\left((e f)^{n}\right)^{*}$ and similarly $f \leq\left((e f)^{n}\right)^{*}$. Using Theorem 2, we have that both $e$ and $f$ are maximal elements of $S$ and therefore $e=\left((e f)^{n}\right)^{*}=f$.

We recall that the natural order, $\leq_{n}$, on the idempotents of a regular semigroup is defined by

$$
e \leq_{n} f \Leftrightarrow e=e f=f e
$$

and that an ordered regular semigroup $(T, \leq)$ is said to be naturally ordered if the order extends the natural order, in the sense that if $e \leq_{n} f$ then $e \leq f$. 


\section{EVENTUALLY POINTED PRINCIPALLY ORDERED REGULAR SEMIGROUPS}

Theorem 4 Let $S$ be an eventually pointed principally ordered regular semigroup. The following statements are equivalent:

(1) $S$ is naturally ordered

(2) $S$ has a biggest idempotent (in fact, a maximal element), $\xi$, and $\xi=e^{*}$ for every idempotent $e$ of $S$.

Proof. (1) $\Rightarrow(2)$ : By [1, Theorem 13.29(3)] we have that, for every idempotent $e$ of $S,\left(e e^{*}\right)^{*}$ is a maximal idempotent. Thus, by Theorem 3, we can conclude that $S$ has a biggest idempotent, $\xi$, which by Theorem 2 is a maximal element of $S$. From (1) and [1, Theorem 13.28] we have that $S$ is a strong Dubreil-Jacotin semigroup and using [1, Theorem 13.20], $e=e(\xi: e) e$ for every idempotent $e$ of $S$. Since, $e \xi \leq \xi$, it then follows that $\xi \leq \xi: e \leq e^{*}$, whence $\xi=e^{*}$, using the fact that $\xi$ is a maximal element of $S$.

(2) $\Rightarrow(1)$ : Let $e$ and $f$ be idempotents of $S$ such that $e \leq_{n} f$, that is $e=e f=f e$. Then, using (2), we have that $e^{*}=\xi=f^{*}$, and therefore

$e=f e f \leq f e^{*} f=f \xi f=f f^{*} f=f$,

from which we conclude that $S$ is naturally ordered.

Note that [4, Theorem 5] states that a pointed principally ordered regular semigroup, $S$, is naturally ordered if and only if it has a biggest element, $\xi$, and $\xi=x^{*}$ for every $x$ in $S$.

Corollary If $S$ is an eventually pointed principally ordered and naturally ordered regular semigroup, then Green's relations $\mathcal{D}$ and $\mathcal{J}$ coincide.

Proof. Let $x$ be an arbitrary element of $S$. Since $S$ is eventually pointed, there exists a positive integer, $n$, such that $x^{2 n} \leq x^{n}$. By [1, Theorem 13.27] we have that the unary operation $x \rightarrow x^{*}$ is antitone, whence $\left(x^{n}\right)^{*} \leq\left(x^{2 n}\right)^{*}$. From Theorem $4, S$ has a biggest idempotent, $\xi$, which is, in fact, a maximal element of $S$, and therefore $\xi \xi \xi=\xi$ implies $\xi \leq \xi^{*}$ whence $\xi=\xi^{*}$.

Now, using $(\alpha), x^{2 n}=x^{n} x^{n} \leq x^{n}\left(x^{n}\right)^{*} \leq \xi$, whence $\xi=\xi^{*} \leq\left(x^{2 n}\right)^{*}$, and consequently $\xi=\left(x^{2 n}\right)^{*}$, which implies $x^{n} \leq\left(x^{n}\right)^{*} \leq\left(x^{2 n}\right)^{*}=\xi$, from which we obtain $\xi=\xi^{*} \leq\left(x^{n}\right)^{*}$ and therefore $\xi=\left(x^{n}\right)^{*}$. Thus,

$x^{2 n}=x^{2 n}\left(x^{2 n}\right)^{*} x^{2 n}=x^{n} x^{n}\left(x^{n}\right)^{*} x^{n} x^{n}=x^{n} x^{n} x^{n}=x^{3 n}$; that is, $x^{2 n}$ is an idempotent of $S$. Therefore $S$ is a group bound and [5, Theorem 1.2.20] allows us to conclude that Green's relations $\mathcal{D}$ and $\mathcal{J}$ coincide.

In the presence of an identity element, 1, an interesting situation can be described.

Theorem 5. Let $S$ be an eventually pointed principally ordered regular semigroup with an identity element, 1 , and consider $T=\{x \in S \mid 1 \leq x\}$. For every $x \in T$, there exists a positive integer, $n$, such that $x^{n}=\left(x^{n}\right)^{*}=\left(x^{n}\right)^{* *}$ is an idempotent of $S$.

Proof. Since $S$ is eventually pointed, for every $x \in T$ there exists a positive integer, $n$, such that $x^{2 n} \leq x^{n}$. We have that $1 \leq x$ and $1=1 \ldots 1 \leq x \ldots x=x^{n}$ whence, by $\left(P_{4}\right), 1 \leq\left(x^{n}\right)^{* *}$. Then, $\left(x^{n}\right)^{*} \cdot 1 \cdot\left(x^{n}\right)^{*} \leq\left(x^{n}\right)^{*}$; that is, $\left(\left(x^{n}\right)^{*}\right)^{2} \leq\left(x^{n}\right)^{*}$ and therefore, using $(\alpha)$,

$$
\left(x^{n}\right)^{*}=1 \cdot\left(x^{n}\right)^{*} \leq\left(x^{n}\right)\left(x^{n}\right)^{*} \leq\left(x^{n}\right)^{*}\left(x^{n}\right)^{*}=\left(\left(x^{n}\right)^{*}\right)^{2} \leq\left(x^{n}\right)^{*}
$$

which gives $\left(x^{n}\right)^{*}=\left(x^{n}\right)\left(x^{n}\right)^{*}$, an idempotent of $S$. Similarly $\left(x^{n}\right)^{*}=\left(x^{n}\right)^{*}\left(x^{n}\right)$, from which we obtain

$$
x^{n}=x^{n}\left(x^{n}\right)^{*}\left(x^{n}\right)^{* *}\left(x^{n}\right)^{*} x^{n}=\left(x^{n}\right)^{*}\left(x^{n}\right)^{* *}\left(x^{n}\right)^{*}=\left(x^{n}\right)^{*}
$$

allowing us to conclude that $\left(x^{n}\right)^{*}=\left(x^{n}\right)^{* *}$ and, therefore, $x^{n}=\left(x^{n}\right)^{*}=\left(x^{n}\right)^{* *}$.

In the special case where $S$ is a pointed principally ordered regular semigroup, with an identity, 1 , we have that the positive integer in the previous theorem is $n=1$. This means that, for these semigroups, we have $x=x^{*}=x^{* *}$ for every element $x$ in $S$ such that $1 \leq x$. Thus, we obtain the following Corollary of Theorem 5 .

Corollary Let $S$ be a pointed principally ordered regular semigroup with an identity element, 1 , and consider $T=$ $\{x \in S \mid 1 \leq x\}$. For every $x \in T, x=x^{*}=x^{* *}$ is an idempotent of $S$.

Proof. It is an immediate consequence of Theorem 5.

From these results, we therefore obtain as a consequence [4, Theorem 7]:

Theorem 6 Let $S$ be a pointed principally ordered regular semigroup with an identity element, 1. The set $S^{*}=\{x \in S \mid 1 \leq x\}$ is a join semilattice in which $x \vee y=x y$.

Proof. We have, for every $x \in S$, that $x \cdot 1 \cdot x=x^{2} \leq x$, which implies that $1 \leq x^{*}$ and, therefore, $S^{*} \subseteq\{x \in S \mid 1 \leq x\}$. Conversely, for any $x \in\{x \in S \mid 1 \leq x\}$, we obtain, by the Corollary to Theorem 5 , that $x=x^{*}$, which means that $\{x \in S \mid 1 \leq x\} \subseteq S^{*}$. Thus, $S^{*}=\{x \in S \mid 1 \leq x\}$. 
The fact that $S^{*}=\{x \in S \mid 1 \leq x\}$ is a join semilattice in which $x \vee y=x y$ follows, using the same argument as in [4, Theorem 7].

Example 5. In $\operatorname{Mat}_{2} B(0,1)$ of Example $1,\left[\begin{array}{ll}1 & 0 \\ 0 & 1\end{array}\right]$ is the identity element and we have

$$
\left(\operatorname{Mat}_{2} B(0,1)\right)^{*}=\left\{\left[\begin{array}{ll}
1 & 0 \\
0 & 1
\end{array}\right],\left[\begin{array}{ll}
1 & 0 \\
1 & 1
\end{array}\right],\left[\begin{array}{ll}
1 & 1 \\
0 & 1
\end{array}\right],\left[\begin{array}{ll}
1 & 1 \\
1 & 1
\end{array}\right],\left[\begin{array}{ll}
0 & 1 \\
1 & 0
\end{array}\right],\left[\begin{array}{ll}
1 & 1 \\
1 & 0
\end{array}\right],\left[\begin{array}{ll}
0 & 1 \\
1 & 1
\end{array}\right]\right\}
$$

while

$$
\left\{\left[\begin{array}{ll}
a & b \\
c & d
\end{array}\right] \in \operatorname{Mat}_{2} B(0,1) \mid\left[\begin{array}{ll}
1 & 0 \\
0 & 1
\end{array}\right] \leq\left[\begin{array}{ll}
a & b \\
c & d
\end{array}\right]\right\}=\left\{\left[\begin{array}{ll}
1 & 0 \\
0 & 1
\end{array}\right],\left[\begin{array}{ll}
1 & 0 \\
1 & 1
\end{array}\right],\left[\begin{array}{ll}
1 & 1 \\
0 & 1
\end{array}\right],\left[\begin{array}{ll}
1 & 1 \\
1 & 1
\end{array}\right]\right\}
$$

This does not contradict Theorem 6 , since $\operatorname{Mat}_{2} B(0,1)$ is not pointed.

In $[4$, Theorem 8$]$ it is proven that any two-comparable $\mathcal{D}$-related idempotents in a pointed principally ordered regular semigroup are mutually inverse. This heavily depends on the fact that in such a semigroup $x \mathcal{D} y$ if and only if $x^{0}=y^{0}$. This is not necessarily true in eventually pointed principally ordered regular semigroups. To see this, let us present an example.

Example 6. In Mat ${ }_{2} B(0,1)$ of Example 1, we have that $\left[\begin{array}{ll}1 & 0 \\ 0 & 1\end{array}\right]$ and $\left[\begin{array}{ll}0 & 1 \\ 1 & 0\end{array}\right]$ are $\mathcal{D}$ related, but

$$
\left[\begin{array}{ll}
1 & 0 \\
0 & 1
\end{array}\right]=\left[\begin{array}{ll}
1 & 0 \\
0 & 1
\end{array}\right]^{0} \neq\left[\begin{array}{ll}
0 & 1 \\
1 & 0
\end{array}\right]^{0}=\left[\begin{array}{ll}
0 & 1 \\
1 & 0
\end{array}\right]
$$

Although the previous description of Green's $\mathcal{D}$ relation is not true in our situation, we can prove a generalization of $[4$, Theorem 8] to the wider class of ordered regular semigroups with biggest inverses.

Theorem 7. If $S$ is an ordered regular semigroup with biggest inverses such that e and $f$ are comparable idempotents, for which $e^{0}=f^{0}$, then e and $f$ are mutually inverse.

Proof. Let us assume without loss of generality that the idempotents $e$ and $f$ are such that $e \leq f$ and $e^{0}=f^{0}$. Then,

and

$$
e=e e e \leq e f e \leq e f^{0} e=e e^{0} e=e \Rightarrow e=e f e
$$

$$
f e f \leq f f f=f=f f^{0} f=f e^{0} f=f e^{0} e e^{0} f=f f^{0} e e e f^{0} f \leq f f^{0} f e f f^{0} f=f e f \Rightarrow f=f e f
$$

which means that $e$ and $f$ are mutually inverse.

In order to characterise, when an eventually pointed principally ordered regular semigroup is completely simple, we consider $C=\left\{x \in S \mid x^{0}=x^{*}\right\}$ the set of compact elements of $S$.

Theorem 8. Let $S$ be an eventually pointed principally ordered regular semigroup. The following statements are equivalent:

(1) $S$ is completely simple;

(2) $S$ is naturally ordered and $E(S) \subseteq C$;

(3) $S$ has a biggest idempotent (in fact, a maximal element), $\xi$, and $\xi=e^{0}$ for every idempotent $e$ of $S$.

Proof. (1) $\Rightarrow$ (2): If $S$ is completely simple then, since $\leq_{n}$ reduces to equality, $S$ is trivially naturally ordered. We have, by Theorem 4, that $S$ has a biggest idempotent, such that for every idempotent $e$ of $S, \xi=e^{*}$. Now,

and

$$
e^{0}=e^{*} e e^{*}=\xi e \xi
$$

$$
e^{0} e^{0}=\xi e \xi \xi e \xi=\xi e \xi e \xi=\xi e e^{*} e \xi=\xi e \xi=e^{0}
$$

gives that $e^{*}=\xi$ and $e^{0}=\xi e \xi$ are idempotents such that

$$
e^{0} e^{*}=\xi e \xi \xi=\xi e \xi=e^{0}=\xi e \xi=\xi \xi e \xi=e^{*} e^{0}
$$

That is, $e^{0} \leq_{n} e^{*}$ and, since $\leq_{n}$ reduces to equality, $e^{0}=e^{*}$, for every idempotent $e$ of $S$. Therefore, we can conclude that $E(S) \subseteq C$. 


\section{EVENTUALLY POINTED PRINCIPALLY ORDERED REGULAR SEMIGROUPS}

$(2) \Rightarrow(1)$ : Suppose now that $S$ is naturally ordered and $E(S) \subseteq C$. Assume that $e$ and $f$ are idempotents of $S$, such that $e \leq_{n} f$. Thus, in particular, $e \leq f$ and also $e=e f=f e$.

By Theorem 4, $S$ has a biggest idempotent (in fact, a maximal element), $\xi$, such that $e^{*}=\xi=f^{*}$. Since $E(S) \subseteq C$ we have that $e^{0}=f^{0}$, whence, by Theorem 7,e and $f$ are mutually inverse and, using the fact that $e \leq_{n} f$,

$$
f=f e f=e f=e .
$$

Hence, $\leq_{n}$ reduces to equality and $S$ is completely simple.

$(2) \Longrightarrow(3)$ : From Theorem $4, S$ has a biggest idempotent (in fact, a maximal element), $\xi$, and $\xi=e^{*}$ for every idempotent $e$ of $S$. Since $E(S) \subseteq C$, we obtain that $\xi=e^{*}=e^{0}$.

$(3) \Longrightarrow(2)$ : By (3), $\xi$ is a biggest idempotent (in fact a maximal element) of $S$, such that $\xi=e^{0}$ for every idempotent $e$ of $S$. By $\left(P_{3}\right)$, we have that $\xi=e^{0} \leq e^{*}$. Since $\xi$ is a maximal element of $S$, we obtain $\xi=e^{*}=e^{0}$ and, by Theorem $4, S$ is naturally ordered and $E(S) \subseteq C$.

Using Theorem 7, we can prove as in [4, Theorem 9] the following result in an eventually pointed principally ordered regular semigroup, under the weaker conditions of $e$ and $f$ comparable idempotents such that $e^{0}=f^{0}$.

Theorem 9. Let $S$ be an eventually pointed principally ordered regular semigroup. If e and $f$ are idempotents of $S$ such that $e \leq f$ and $e^{0}=f^{0}$, then the subalgebra $T$ of $\left(S,{ }^{*}\right)$ generated by $\{e, f\}$ is a band having at most 10 elements. In the case where $T$ has precisely 10 elements it is represented by the Hasse diagram.

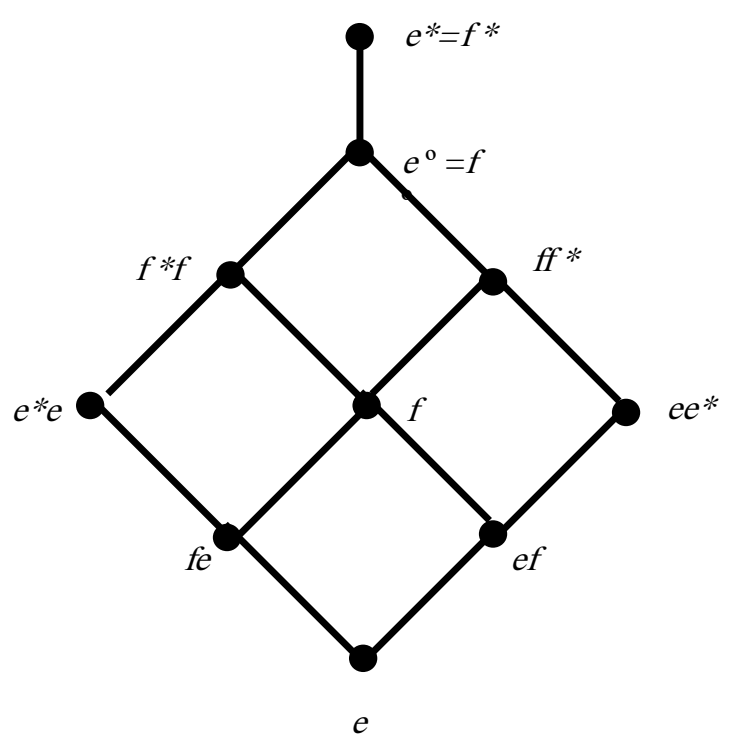

in which elements joined by lines of positive gradient are $\mathcal{R}$-related, those joined by lines of negative gradient are $\mathcal{L}$-related, and the vertical line also indicates $\leq_{n}$.

Example 7. Consider in Example 1

$$
e=\left[\begin{array}{ll}
0 & 0 \\
0 & 1
\end{array}\right] \quad \text { and } \quad f=\left[\begin{array}{ll}
0 & 1 \\
0 & 1
\end{array}\right]
$$

that verify $e \leq f$ and $e^{0}=\left[\begin{array}{ll}1 & 1 \\ 1 & 1\end{array}\right]=f^{0}$. This gives a simplified version of the structure presented in Theorem 9 , the four element set, with the induced order and matrix multiplication,

$$
T=\left\{\left[\begin{array}{ll}
0 & 0 \\
0 & 1
\end{array}\right],\left[\begin{array}{ll}
0 & 1 \\
0 & 1
\end{array}\right],\left[\begin{array}{ll}
0 & 0 \\
1 & 1
\end{array}\right],\left[\begin{array}{ll}
1 & 1 \\
1 & 1
\end{array}\right]\right\}
$$

To obtain, precisely, the diagram in Theorem 9, let us recollect that in [4, Example 4] we obtained this diagram, in a pointed principally ordered regular semigroup, with a semigroup denoted by $E\left(B_{2}\right)$. If we define, in the Cartesian ordered set, $T \times E\left(B_{2}\right)$, the Cartesian multiplication, we easily obtain an eventually pointed principally ordered regular semigroup with a copy of the diagram in Theorem 9. We just need to consider, for example, the Cartesian product, Cartesian order of $\{e\}$ by the band obtained in [4, Example 4]. 


\section{PINTO, G.A.}

\section{Conclusion}

In this paper we introduced a new class of ordered semigroups: Eventually Pointed Principally Ordered Regular Semigroups, as a generalisation of pointed principally ordered regular semigroups. Examples showing that the classes are distinct were presented, and it was possible to prove several of the known results, or similar versions of them, in this class of semigroups, with new and adjusted justifications.

\section{Conflict of interest}

The author declares no conflict of interest.

\section{Acknowledgement}

I thank Sultan Qaboos University for providing facilities.

\section{References}

1. Blyth, T.S. and Pinto, G.A. Principally ordered regular semigroups, Glasgow Mathematics Journal. 32 (1990) 349-364. doi:10.1017/S0017089500009435.

2. Blyth, T.S. and Pinto, G.A. Idempotents in principally ordered regular semigroups, Communications in Algebra 19 (1991) 1549-1563. doi:10.1080/00927879108824220.

3. Blyth, T.S. Lattices and Ordered Algebraic Structures, (Springer 2005). doi:10.1007/b139095.

4. Blyth, T.S. and Pinto, G.A. Pointed principally ordered regular semigroups, Discussiones Mathematicae 36 (2016) 101-111. doi:10.7151/dmgaa.1243.

5. Higgins, P.M. Techniques of Semigroup Theory (Oxford Science Publications, 1992) doi:10.1007/BF02573500.

Received 29 May 2019

Accepted 2 September 2019 